\title{
An adaptive relay node selection algorithm based on opportunity
}

\author{
Baoju Zhang ${ }^{*}$, Cuiping Zhang, Jiasong Mu, Wei Wang and Jiazu Xie
}

\begin{abstract}
The collaborative relay network plays an important role in a smart grid application thanks to its high efficiency. However, energy and bandwidth management need to be further improved to extend network life cycle and reduce potential network segmentation. Aiming to balance the network energy consumption, this paper proposes an adaptive relay node selection algorithm based on the opportunity (OAR). In OAR, the potential relay nodes are chosen to act as candidates based on bit error rate (BER) estimation, and then, the relay is adaptively and opportunistically determined for packet forwarding. Residual energy is considered to avoid fast energy exhaustion for some certain nodes. The simulation results show that the proposed OAR algorithm improves the network performance by deferring the earliest death time of the nodes and extending the network life cycle.
\end{abstract}

Keywords: Collaborative relay, BER, Adaption, Energy consumption, Life cycle, Wireless smart grid

\section{Introduction}

Smart grid plays an important role in the national energy industry chain. Nowadays, a communication system called wireless smart grid plays an irreplaceable role in real life.

The smart grid is a wireless hybrid network, which is supported by a based station of a wired network (intelligent monitoring system) to assist remote communication (https://en.wikipedia.org/wiki/Smart_grid) among wireless nodes (smart meters and sensors). Wireless smart grid is composed of $\mathrm{n}$ wireless nodes, which lack support from a fixed infrastructure. Due to the lack of a fixed infrastructure support, data transmission cannot be transmitted through cable facilities, instead of being transferred to the destination node in a multi-hop way.

When a node in the wireless smart grid is scheduled to transmit data, nodes will be selected from the same cell to cooperate with the source node. This form of transmission can be achieved as long as this transmission does not damage the transmission rate [1]. These nodes are called the source of opportunity. Then, it will be a worthy question that what can become a source of opportunity to assist the source node to complete the data transmission more effectively. The source of opportunity mentioned here is the

\footnotetext{
* Correspondence: tjnuzcp2@163.com; wdxyzbj@163.com Tianjin Key Laboratory of Wireless Mobile Communications and Power Transmission, Tianjin Normal University, Tianjin 300387, China
}

candidate relay nodes in this article. Due to the limited node resources, the traverse method is not able to effectively achieve low enough lantency which is crucial in smart grid application is restricted, and due to the high standard of node communication processing and communication delay, using the traverse method to select the relay node does not work effectively.

The research of classical cooperative relay communication technology has focused on the improvement of system performance, but takes no account of the energy efficiency of the system. At present, from an energy efficiency stance, the literature $[2,3]$ demonstrated that the energy consumption of the wireless communication network can be improved by cooperative diversity techniques. P. Rost used a fixed relay structure in the cellular communication which could reduce the energy consumption effectively and thus enhance system performance in case of increasing the density of the relay [4]. Nokleby and Aazhang used the game theory model to demonstrate that the transmission energy efficiency of two users could be improved under different channel conditions by a collaboration of two users [5]. But related researches above do not take into account the average energy consumption of all nodes and selecting relay nodes adaptively based on opportunity, which thus extends the life cycle of the network.

This paper proposes an adaptive relay node selection algorithm based on opportunity with the purpose of 
balancing node energy consumption, making the node to be more efficient and working in longer hours, and improving the performance of wireless smart grid system, which can effectively and greatly achieve a balance of network energy consumption and extend the life cycle of the wireless smart grid system. The rest of this paper is organized as follows: In Sections 2 and 3, the author will introduce the system model and OAR selection algorithm. Section 4 contains the experimental evaluation of the proposed algorithm. Section 5 concludes the whole paper.

\section{System model}

Considering its own characters of the wireless smart grid, such as wireless channel multipath fading, path loss, shadow effects, and other unfavorable factors, the direct communication efficiency between the source node and the destination node is not high [6]. We assume that wireless sensor cooperative communication network contains $\mathrm{n}$ sensor nodes and a destination node, in which $\mathrm{n}$ sensor nodes can work as a source node to send data, and also function as a relay node to forward data, as is shown in Fig. 1. We assume that the relay node is selected from the candidate relay nodes. According to the requirement of the bit error rate (BER) threshold, we select the relay node that BER is lower to assist the source node to send information to the destination node. The BER threshold is the direct link BER.

In this system, besides the channel between the source node $(S)$ and destination node $(D)$, there also exist channels between the source node and each relay node $(R)$ and each relay node and destination node. $d_{s d}$ represents the distance from $S$ to $D, d_{s r_{i}}$ between $S$ and $R$, and $d_{r_{i} d}$ between $R$ and $D$. In the model above, the entire relay process can be divided into two time slots [7].

In time slot $1, S$ sends the information data to $R$ and $D$, then the received signals $y_{s, r}(t)$ and $y_{s, d}(t)$ come, respectively, from $R$ and $D$ :

$$
\begin{aligned}
& y_{s, r}(t)=\sqrt{P_{A}} h_{s, r} x(t)+n_{s, r} \\
& y_{s, d}(t)=\sqrt{P_{A}} h_{s, d} x(t)+n_{s, d}
\end{aligned}
$$

$P_{A}$ is the transmission power of $S, h_{s, r}$ and $h_{s, d}$ are the channel coefficients of $S$ to $R$ and to $D$, and $n_{s, r}$ and $n_{s, d}$ are the corresponding Gauss white noise.

In time slot $2, R$ will forward the message to $D$, at which $D$ receives the signal $y_{r, d}(t)$ from the relay node that is given:

$$
y_{r, d}(t)=\sqrt{P_{R}} h_{r, d} x(t)+n_{r, d}
$$

$P_{R}$ means the transmission power of $R, h_{r, d}$ represents the channel coefficient of $R$ to $D$, and $n_{r, d}$ shows the corresponding additive Gauss white noise. Without loss of generality, we suppose that $n_{s, n} n_{s, d}$, and $n_{r, d}$ are subject to a Gaussian distribution with a mean of zero and a variance of $N_{0}$. If the relay node adopts the AF relaying mode, then there is

$$
y_{r, d}(t)=\beta h_{r, d} y_{s, r}(t)+n_{r, d}
$$

$\beta$ is the relay amplification factor. If $D$ adopts the maximum ratio combination, the received signal of the destination node is

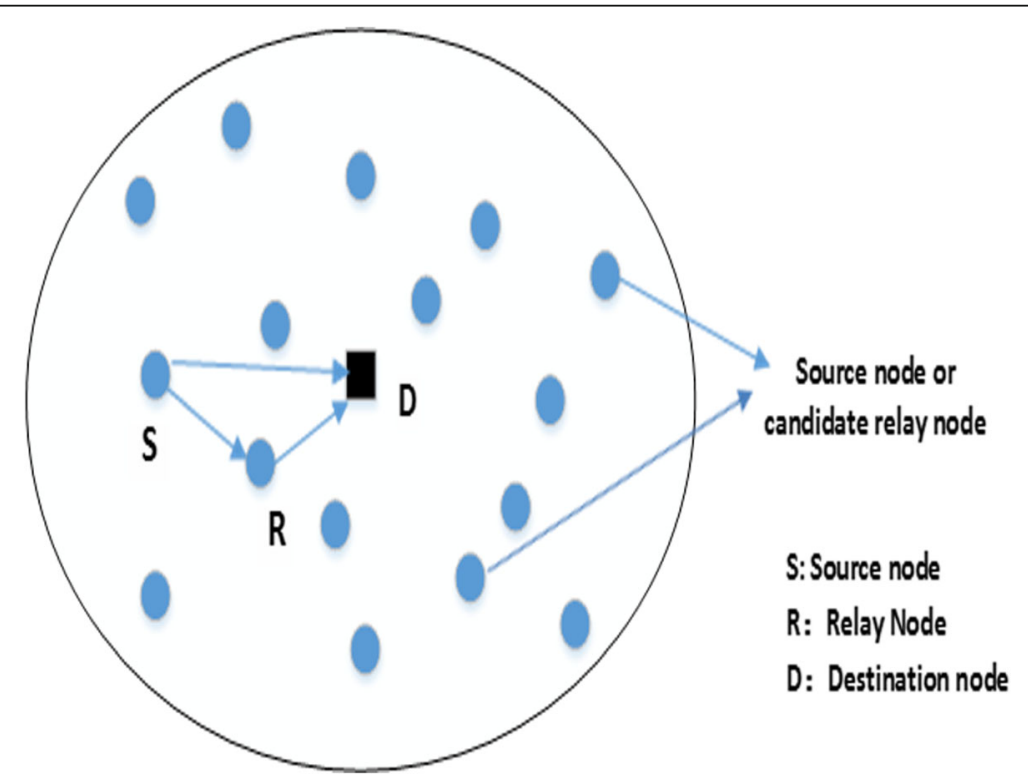

Fig. 1 System model of collaborative relay transmission 


$$
y(t)=\omega_{1} y_{s, d}(t)+\omega_{2} y_{r, d}(t)
$$

$\omega_{1}$ and $\omega_{2}$ are weighted factors. This paper assumes that the transmission adopts the BPSK modulation method.

\section{An adaptive relay node selection algorithm based on opportunity for wireless smart grid system}

\subsection{Candidate relay node set selection method}

The OAR selection algorithm is considered from the goal of meeting the minimum BER requirement. That is to say, the BER of the data transmission from $S$ to $D$ is calculated firstly, and set it as the threshold for selecting candidate relay nodes. Then, the relay node calculates the average BER at the time of forwarding data, and automatically determines whether the average BER is less than the BER threshold. If so, these relay nodes constitute a set of candidate relay nodes that assist the source node to complete data transmission efficiently.

In AF relay mode, the SNR (signal to noise ratio) of the receiver with its maximum ratio is

$$
\gamma_{d}=\gamma_{s, d}+\sum \frac{\gamma_{s, r} \gamma_{r, d}}{\gamma_{s, r}+\gamma_{r, d}}+1
$$

$\gamma_{s, d}=P_{A}\left|h_{s, d}\right|^{2} / N_{0}$ represents the SNR of the direct link, $\gamma_{s, r}=P_{A}\left|h_{s, r}\right|^{2} / N_{0}$ represents the receiver SNR of $R$, $\gamma_{r, d}=P_{R}\left|h_{r, d}\right|^{2} / N_{0}$ represents the receiver SNR of $D$ from $R$.

Under the circumstances of high SNR, 1 in formula (4) can be ignored and be approximated as

$$
\gamma_{d}=\gamma_{s, d}+\sum \frac{\gamma_{s, r} \gamma_{r, d}}{\gamma_{s, r}+\gamma_{r, d}}
$$

According to the basic knowledge of communication theory, the BER formula based on SNR can be expressed as $P_{e}=Q \sqrt{k \gamma_{d}}$. The constant $k$ is related to the modulation mode. In binary phase shift keying, the value of $K$ is $2, Q(x)=1 / \sqrt{2 \pi} \int_{x}^{+\infty} e^{-\frac{t^{2}}{2}} d t[8]$.

In this way, the BER formula can be used to calculate the direct transmission link. That is, the BER of $S$ to $D$ is

$$
P_{e}(d)=Q \sqrt{k \gamma_{d}}
$$

Similarly, we can calculate the BER from $S$ to the candidate relay node $R_{i}$, that is $P_{e}\left(S, R_{i}\right)=Q\left(\sqrt{k \gamma_{s, r}}\right)$. The candidate relay node has two kinds of cases: correct reception and incorrect reception.

When the candidate relay node $R_{i}$ receives correctly, with the help of a single $R_{i}$, the BER from $S$ to $D$ can be expressed as $P_{e}\left(S, R_{i}, D\right)=Q\left(\sqrt{k\left(\gamma_{s, d}+\gamma_{r, d}\right)}\right)$.

When the candidate relay node $R_{i}$ receives incorrectly, it does not transmit power, which means that it does not assist in transmitting information. At this time, the BER between $S$ and $D$ can be expressed as $P_{e}(S, D)=Q$ $\left(\sqrt{k \gamma_{s, d}}\right)$. Thus, when a single relay node $R_{i}$ is selected, its average BER can be expressed as

$$
P_{e}(i)=\left(1-P_{e}\left(S, R_{i}\right)\right) * P_{e}\left(S, R_{i}, D\right)+P_{e}\left(S, R_{i}\right) * P_{e}(S, D)
$$

In this OAR selection algorithm, the relay nodes that meet the requirement of BER $P_{e}(i)<P_{e}(d)$ making up the candidate relay nodes assist the source node to complete the efficient transmission of data together.

\subsection{Analysis of node energy consumption and node survival rate in wireless smart grid}

Wireless smart grid scale is usually very large, while the node's energy supply and hardware resource are limited. It is very difficult to replace or recharge the battery after the node has been exhausted. According to different application requirements, there are many definitions of the network life cycle, which usually refers to the duration time that starts from the beginning of the network to the point that it cannot complete the requirement of the application. Due to the limitation of the node's energy and its difficulty of replacement, the network life cycle depends on the time when the first network node dies. The later the first dying node occurs, the longer the life cycle of the network is.

Most of the destination nodes in the wireless smart grid are far away from the source node. Direct communication can cause a lot of energy waste. Therefore, it is necessary to construct a multi-hop transmission between nodes to reduce the node energy consumption and that of the whole system according to the characteristics of the wireless smart grid. For the wireless smart grid, the OAR selection algorithm proposed in this paper can balance the energy consumption of the relay nodes, which can enable the node to work more efficiently and in a longer working time, consequently prolonging the life cycle of the whole wireless smart grid system.

The energy consumption of sensor nodes can be categorized into three parts [9]: the transmission energy consumption generated by the data transmission is $E_{t}$, the received energy consumption generated by the data reception is $E_{n}$ and the perceived energy consumption generated by the sensing data is $E_{p}$. Among them, $E_{t}$ and $E_{r}$ constitute the main part of energy consumption. According to the article [10], transmission energy consumption generated by the transmission of $k$-bit data from node $i$ to node $j$ can be defined as: 


$$
E_{t}=\left\{\begin{array}{l}
k E_{\text {elec }}+k \varepsilon_{f s} d^{2}, \text { if } d<d_{0} \\
k E_{\text {elec }}+k \varepsilon_{\text {amp }} d^{4}, \text { if } d \geq d_{0}
\end{array}\right.
$$

The value of $E_{t}$ depends on the transmission distance from node $i$ to node $j$ and the amount of data being transmitted. If $d$ is smaller than the threshold value $d_{0}$, the transmission loss will use the free space mode; accordingly, if $d$ is greater than the threshold value $d_{0}$, the transmission loss is chosen for the multipath model. $E_{\text {elec }}$ is the electrical energy that is related to digital coding and other factors, $\varepsilon_{f s}$ is the amplification energy that is related to the sensitivity of receiver, and $\varepsilon_{a m p}$ also is amplification energy that is related to the received noise image. The received energy consumption $E_{r}$ can be defined as

$$
E_{r}=k E_{\text {elec }}
$$

Under restricted conditions:

(1) $d<d_{0}$

(2)When the node functions as the source node, and the initial energy is $E_{0}$, which sends $k$-bit data, the transmission energy consumption of $S$ is

$$
E_{s}=k E_{\text {elec }}+k \varepsilon_{f s} d_{s r_{i}}^{2}
$$

The residual energy of the source node is

$$
E_{s, r e}=E_{0}-E_{s}
$$

(3) When the node functions as the relay node, the initial energy is $E_{0}$. The relay node $R_{i}$ receives the $k$ bit valid data, and then forwards the $k$-bit valid data to the destination node. $d_{r_{i} d}(i \in[1, N])$ is the distance between the relay node and destination node, then the total energy consumption of $R$ is

$$
\begin{aligned}
E_{i, \text { total }} & =k E_{\text {elec }}+k \varepsilon_{f s} d_{r_{i} d} d^{2}+k E_{\text {elec }} \\
& =2 k E_{\text {elec }}+k \varepsilon_{f_{s}} d_{r_{i} d}{ }^{2}
\end{aligned}
$$

The residual energy of the relay node is

$$
E_{i, r e}=E_{0}-E_{i, t o t a l}
$$

(4) The energy of $D$ is not limited

\subsection{An adaption relay node selection algorithm based on opportunity for wireless smart grid system}

This paper presents the opportunity (OAR) selection algorithm, which is described as follows. To meet the communication reliability requirement, the algorithm calculates the BER from $S$ to $D$ firstly, then, sets it as the threshold for selecting the candidate relay nodes. Secondly, the relay node calculates the average BER at the time of forwarding data and automatically determines whether the average BER is less than the BER threshold. We will select the relay node that meets the threshold requirement to form the candidate relay node set. Thirdly, we select the relay node adaptively from the candidate relay node set to forward data.

The relay node compares its residual energy of forwarding data with its initial energy. If the residual energy is $10 \%$ more of its initial energy of the node, the system adaptively selects the relay node to transmit data depending on the probability that the node is selected; if the residual energy is $10 \%$ less of the initial energy of the node, the node dies. If the percentage of dead nodes is greater than $30 \%$, the entire network life cycle will come to an end.

In order to select the relay node to transmit data from the candidate relay nodes set, the probability that node $i$ is selected as the relay node based on the residual energy of the node is

$$
p(i)_{\text {Selected }}=\frac{E_{i, r e} / E_{s, r e}}{\sum_{i=1}^{N} E_{i, r e}+\sum_{r=1}^{N} E_{s, r e}}
$$

When the residual energy of the candidate relay node is enormous, then the probability of being selected as a relay node is high. In this paper, the relay node $M^{*}$ with the maximum value of $p(i)_{\text {Selected }}$ is selected as the relay node to transmit data, that is,

$$
M^{*}=\underset{i}{\arg }\left(\max \left(P(i)_{\text {Selected }}\right)\right)
$$

The specific steps of OAR selection algorithm are as follows:

(1)According to formula (6), calculating the BER threshold of candidate relay nodes

(2) According to formula (7), selecting relay nodes that meet the BER threshold requirement to form a candidate relay node set

(3) According to formula (14), selecting relay nodes adaptively depending on node residual energy from the candidate relay node set to assist the source node to complete data transmission.

(4) According to formulas (11) and (13), determining whether the node is dead

(5)If the node energy consumption is $10 \%$ less of its initial energy of the node, the node is dead. When the percentage of dead nodes is greater than $30 \%$, the entire network life cycle will come to an end

The flow chart of the OAR selection algorithm proposed in this paper is shown in the Fig. 2. In this paper, such a cycle is called a round number or a wheel. 


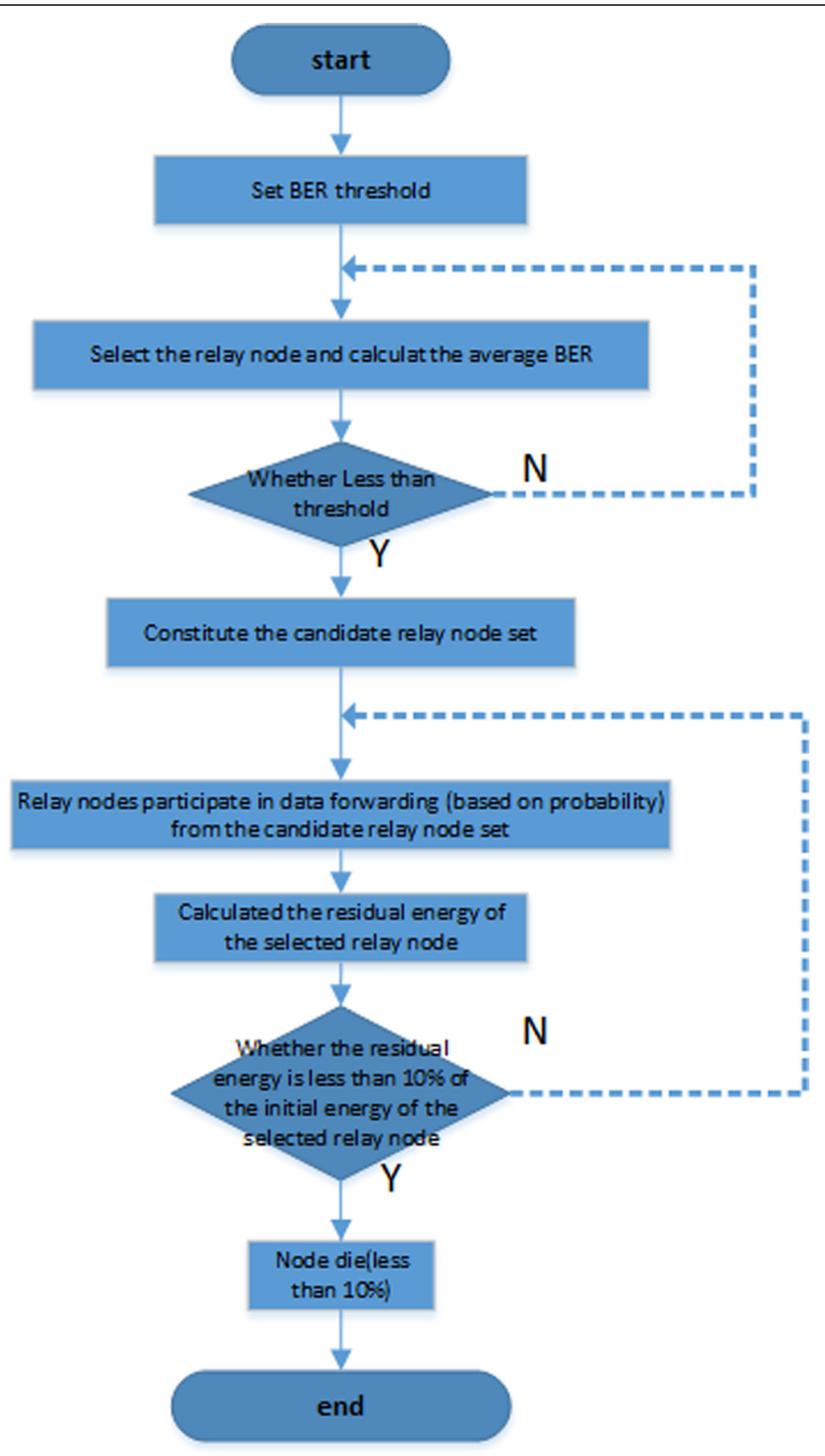

Fig. 2 The flow chart of OAR selection algorithm

\section{Simulation experiments and results analysis}

In order to verify and evaluate the performance of OAR selection algorithm proposed in this paper, we simulate the OAR selection algorithm in this paper. Before the analysis, we make the following assumptions and regulations. Simulation conditions: once the nodes in the network are deployed, they are still stationary; channels between nodes are Rayleigh flat fading channel, the fading between channels is independent, and each channel noise is an additive Gaussian white noise (AWGN) with a mean of 0 and a variance of 1 ; each node works in a half-duplex mode; the node power is distributed averagely and $P_{A}=P_{R} ;$ OAR selection algorithm is used to select relay note and transmit data; we assume that the network consists of 100 nodes that meet the requirements of the BER (which can be used as a source node 
Table 1 Definitions of parameters and variables used in this article

\begin{tabular}{lllll}
\hline Parameter & $E_{\text {elec }}(n / / b i t)$ & $\varepsilon_{f_{5}}\left(p J / b i t / m^{2}\right)$ & $E_{0}(J)$ & $k(b t)$ \\
\hline Value & 50 & 10 & 0.5 & 4000 \\
\hline
\end{tabular}

or a relay node) and a destination node; and when the residual energy of the node is $10 \%$ less than the initial energy of the node, it is considered that the node is dead. When the percentage of dead nodes is more than $30 \%$, the life cycle of the whole system in the network will also come to an end.

The experimental parameters are shown in Table 1. According to the average data of several experiments and the parameter values of the classical experiments, the empirical parameter values of the algorithm are obtained. A comparison can be made on the direct transmission and the minimum energy consumption transmission as well as OAR selection algorithm. Direct transmission, as the name implies, $S$ transfers data to $D$ directly. The minimum energy consumption of the relay node is selected to do the comparison in this paper.

\subsection{Number of dead nodes}

In order to verify the validity of the proposed OAR selection algorithm, the number of network dead nodes of three algorithms is compared under the same initial energy. As shown in Fig. 3, since the direct transmission is not taken into account of the adaptive adjustment process of the node based on the opportunity, the number of dead nodes in the direct transmission is more than the minimum energy consumption transmission and OAR selection algorithm transmission. At the same time, the minimum energy consumption transmission only takes the minimum energy consumption standard to select relay node, it does not count the average energy consumption of all nodes, thus accelerating the death time of the first node.

As can be seen from the figure, in the first 400 rounds, the three algorithms did not appear to be dead nodes; 400 rounds after, the dead nodes began to appear. The OAR selection algorithm's first dead node appears at the latest, the time of the first death of the node that appears on the OAR selection algorithm is about one time higher than that of the direct transmission, and the OAR selection algorithm extends the time of the first dead node, 59.8\% compared with the minimum energy consumption transmission.

\subsection{Network life cycle}

The network lifecycle shows the time that the network can work properly. There are many definitions of the life cycle of the network, but the duration time from the beginning until the dead node percentage is greater than $30 \%$ which is defined as the network life cycle in this paper. In order to ensure the reliability of the experimental results, three algorithms were carried out in 10 experiments, and we calculate the average life cycle of the 10 experiments, and the results are shown in Fig. 4. As can be seen from the figure, the OAR selection algorithm can extend the network life cycle greatly. When the number of candidate nodes is 200, the life cycle of the OAR selection algorithm is about 0.92 times higher than that of the direct transmission, and the network life

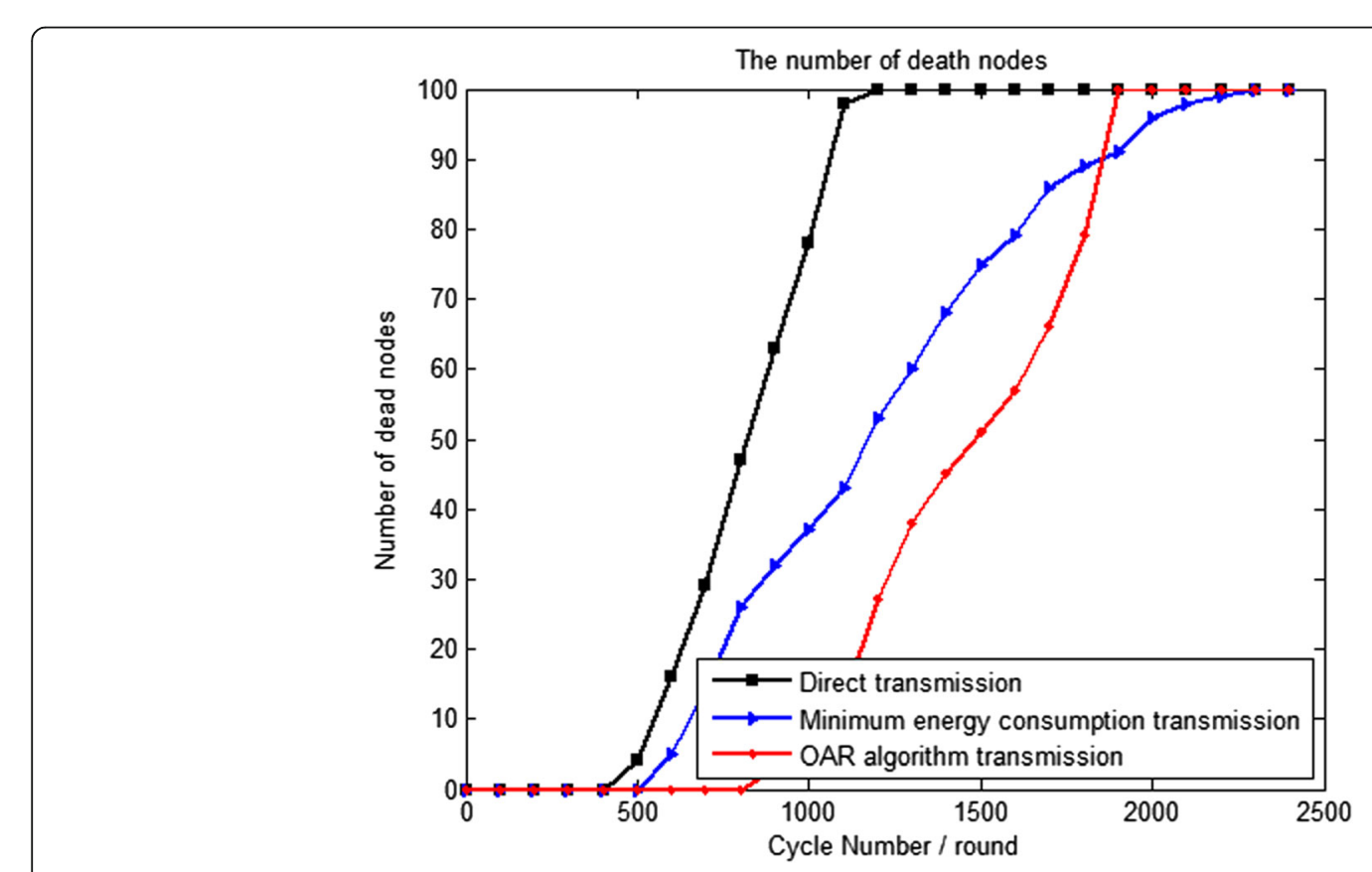

Fig. 3 The number of dead nodes 


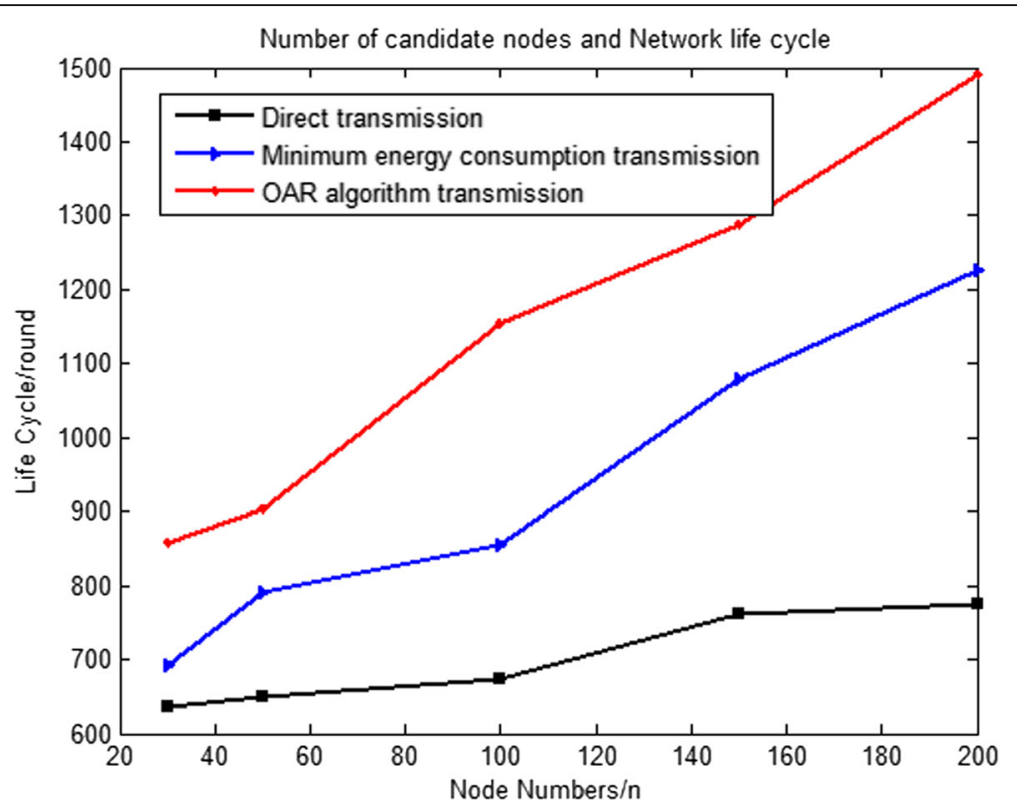

Fig. 4 Number of candidate nodes and network life cycle

cycle is extended by $21.4 \%$ than the minimum energy consumption transmission.

\subsection{Network energy consumption}

The network energy consumption consists of the energy consumption from $S$ to $R$ and the energy consumption of $S$ to $R$. Figure 5 shows the total energy consumption curve of the network nodes of three algorithms. As shown in Fig. 5, the network total energy consumption of direct transmission is higher than the minimum energy consumption transmission, and these two algorithms are higher than the OAR selection algorithm. Moreover, the energy consumption curve of the OAR selection algorithm shows a slight change which suggests that a relay node selected by an OAR selection algorithm has the ability of balancing node energy consumption, thus minimizing the total energy consumption of the network and prolonging the life of the network effectively.

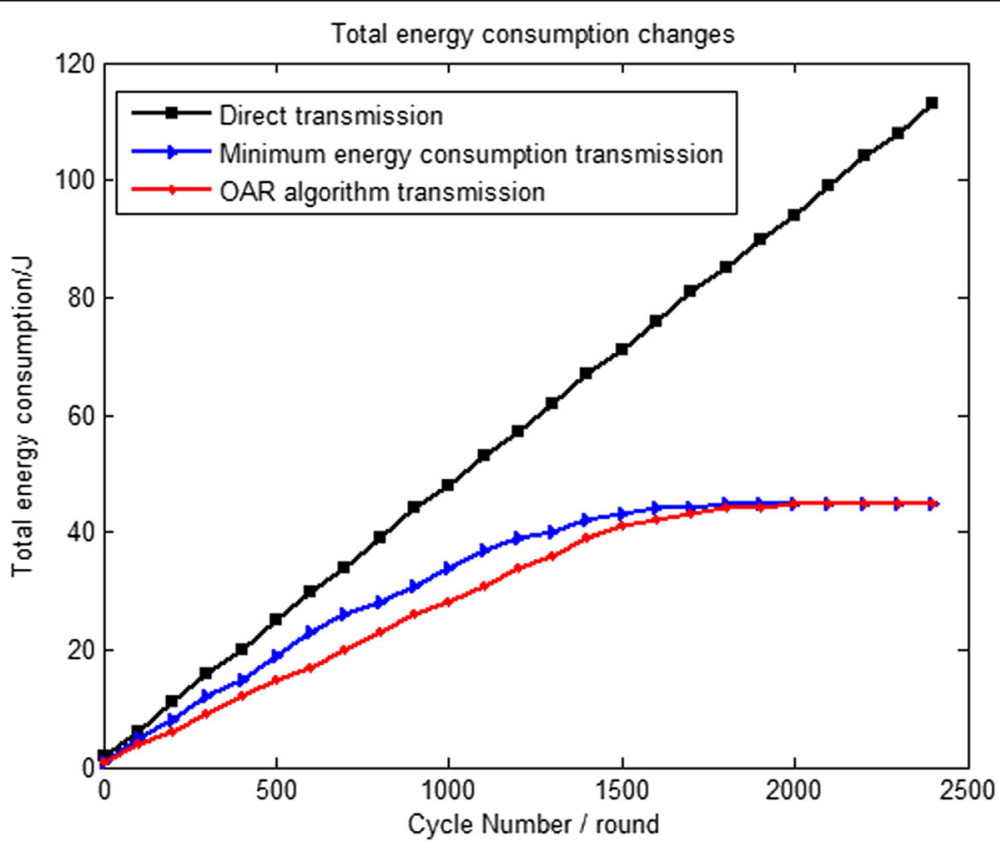

Fig. 5 The total energy consumption of the network node 


\section{Conclusions}

The relay node selection algorithm extends the life cycle of the network to a certain extent. But if we select the optimal location relay selection algorithm to transmit data, the relay node may die prematurely due to a heavy load. If we choose the minimum energy consumption transmission, it will also accelerate the death of the node with low residual energy without thinking of the energy consumption of the source node. This paper presents an adaptive relay node selection algorithm based on the opportunity (OAR) that contrasts the number of dead nodes and the network life cycle, the total energy consumption of direct transmission, and the minimum energy consumption transmission. It is found that the proposed OAR algorithm can improve the network performance by deferring the earliest death time of the nodes, balancing the energy consumption of each node, and extending the network life cycle.

\section{Acknowledgements}

This paper is supported by the Natural Science Foundation of China (61271411) and the Natural Youth Science Foundation of China (61501326, 61401310). It also supported by Tianjin Research Program of Application Foundation and Advanced Technology (15JCZDJC31500) and Tianjin Science Foundation (16JCYBJC16500).

\section{Competing interests}

The authors declare that they have no competing interests.

\section{Publisher's Note}

Springer Nature remains neutral with regard to jurisdictional claims in published maps and institutional affiliations.

Received: 23 March 2017 Accepted: 10 May 2017

Published online: 30 May 2017

\section{References}

1. X Wang, Q Liang, On the throughput capacity and performance analysis of hybrid wireless networks over fading channels. IEEE Trans. Wirel. Commun. 12(6), 2930-2940 (2013)

2. L Simic, SM Berber, KW Sowerby, Partner choice and power allocation for energy efficient cooperation in wireless sensor networks. (Proceedings of IEEE International Conference on Communications, ICC, Beijing, 2008), pp. 42554260

3. Z Zhou, S Zhou, JH Cui, S Cui, Energy-efficient cooperative communication based on power control and selective single-relay in wireless sensor networks. IEEE Trans. Wirel. Commun. 7(8), 3066-3078 (2008)

4. P Rost, G Fettweis, Cooperative Cellular Wireless Networks (Cambridge University Press, Cambridge, 2010), pp. 300-323

5. M Nokleby, B Aazzhang, User cooperation for Energy-Efficient Cellular Communication, (Proceedings of IEEE International Conference on Communications, ICC, Cape Town, 2010), pp.1-5

6. O Simeone, I Stanojev, S Savazzi, Y Bar-Ness, U Spagnolini, Spectrum leasing to cooperating secondary ad hoc networks. IEEE J. Sel. Areas Commun. 26(01), 203-213 (2008)

7. LI Yi, L Qiu, WP Liu, Multi-relays selection scheme in cooperative networks based on decode-and-forward protocol. Commun. Technol. 43(04), 56-58 (2010)

8. A Ribeiro, X Cai, GB Giannakis, Symbol error probabilities for general cooperative links. IEEE Trans. Wirel. Commun. 4(3), 1264-1273 (2005)

9. S Olariu, I Stojmenovic, Design guidelines for maximizing lifetime and avoiding energy holes in sensor networks with uniform distribution and uniform reporting. (Proceedings of IEEE INFOCOM, Barcelona, Catalunya, 2006), pp. 1-12

10. WB Heinzelman, AP Chandrakasan, H Balakrishnan, 2002. IEEE Trans. Wirel. Commun. 1(4), 660-670 (2002)

\section{Submit your manuscript to a SpringerOpen ${ }^{\circ}$ journal and benefit from:}

- Convenient online submission

- Rigorous peer review

- Open access: articles freely available online

- High visibility within the field

- Retaining the copyright to your article

Submit your next manuscript at $>$ springeropen.com 